\title{
Femtosecond Microjoule-Class Ytterbium Fiber Lasers
}

\author{
C. Lecaplain ${ }^{1}$, B. Ortaç ${ }^{2}$, G. Machinet ${ }^{3}$, J. Boullet ${ }^{3}$, M. Baumgartl ${ }^{4}$, T. Schreiber ${ }^{5}$, E. Cormier $^{3}$, A. Hideur ${ }^{1}$ \\ 1. CNRS UMR 6614 CORIA, Université de Rouen, Avenue de l'université BP 12, 76801 Saint Etienne du Rouvray Cedex, France \\ 2. UNAM-Institute of Materials Science and Nanotechnology, Bilkent University, 06800 Bilkent, Ankara, Turkey \\ 3. Université de Bordeaux-CNRS-CEA, CELIA, 351 cours de la Libération F-33405 Talence, France \\ 4. Institute of Applied Physics, Friedrich Schiller University Jena, Albert-Einstein-Str. 15, 07745 Jena, Germany \\ 5. Fraunhofer Institute for Applied Optics and Precision Engineering, Albert-Einstein-Str. 7, D-07745 Jena, Germany \\ lecaplain@coria.fr
}

\begin{abstract}
We report the generation of $830 \mathrm{~nJ}$ energy from a mode-locked all-normal dispersion fiber laser featuring large-mode-area photonic crystal fibers. After external compression, $550 \mathrm{fs}$ pulses with 1.2 MW peak power are demonstrated.

OCIS codes: (140.3510) Lasers, fiber, (140.7090) Ultrafast lasers, (060.5530) Pulse propagation and solitons.
\end{abstract}

\section{Introduction}

High-power femtosecond laser sources are versatile tools for numerous applications ranging from material processing on a sub-micrometre scale to high-field physics. Developing compact and robust oscillators of high energy femtosecond pulses has therefore generated strong research interest during the past decade leading to significant advances in the field. In particular, impressive performance levels, with microjoules energies and up to hundred watts average powers, have been achieved with thin-disk lasers based on Yb-doped crystals [1]. Another promising solution for energy scaling in mode-locked oscillators is rare-earth-doped fibers. Fiber-based sources exhibit very high gain per pass, excellent thermo-optical properties and high mechanical stability making them very suitable for high-power applications. The fundamental challenge for ultrafast fiber lasers relies on the control of the excessive nonlinearity which hinders a self-consistent pulse evolution at high-energy levels. To some extent, the pulse energy can be scaled by increasing the amount of net positive cavity dispersion, which tends to scale down the peak power inside the fiber core by stretching the pulse during its propagation. This is the principle underlying stretched-pulse [2] and similariton lasers [3]. More recently, new routes for energy scaling of mode-locked fiber oscillators have been opened with the development of all-normal dispersion fiber lasers [4-5]. In order to achieve self-consistent pulse evolution, such lasers need a strong pulse shaping mechanism which could be provided by a passive spectral filter [5] or by combination of self-amplitude modulation with gain filtering [4]. Moreover, the employment of low-nonlinearity large-mode-area photonic crystal fibers (PCF) enables significant power scaling. This has been demonstrated recently in all-normal dispersion laser configurations using different pulse shaping mechanisms [6-8]. Notably, the extension of this approach to photonic crystal rods opens the road for subpicosecond microjoule-class fiber sources [9-10]. In this communication, we report the generation of high-energy sub-picosecond pulses from a highly normal dispersion fiber laser featuring a Yb-doped rod-type photonic crystal fiber and a large-mode-area photonic crystal fiber. The use of a long passive fiber allows controlling the total net cavity dispersion. Preliminary results show that $13 \mathrm{~W}$ of average power at $15.5 \mathrm{MHz}$ repetition rate, corresponding to more than $830 \mathrm{~nJ}$ pulses.

\section{Experimental setup}

The experimental setup of the passively mode-locked fiber oscillator is shown in Fig.1. The gain fiber consists in a $95 \mathrm{~cm}$ long $\mathrm{Yb}$-doped photonic crystal fiber with a single-mode core diameter of $80 \mu \mathrm{m}$. The laser cavity is mounted in sigma-configuration around a polarization-sensitive isolator. The large-mode-area photonic crystal fiber is cladding-pumped with a fiber-coupled laser diode emitting $50 \mathrm{~W}$ at $976 \mathrm{~nm} \mathrm{~A} 7 \mathrm{~m}$ long passive LMA microstructure fiber with a core diameter of $25 \mu \mathrm{m}$ is inserted inside the cavity, leading to a total cavity dispersion of about 0.152 $\mathrm{ps}^{2}$. Insertion of the passive fiber after the output coupling allows controlling both the dispersion and the accumulated nonlinear phase along the cavity. Passive mode locking is achieved using a fast saturable absorber mirror (SAM). The SAM has a low-intensity absorption of $30 \%$, a modulation depth of $20 \%$, and a saturation fluence of $120 \mu \mathrm{J} / \mathrm{cm}^{2}$ with a relaxation time of $<500 \mathrm{fs}$. The anti-resonant design of the SAM structure ensures an absorption bandwidth of more than $45 \mathrm{~nm}$. 


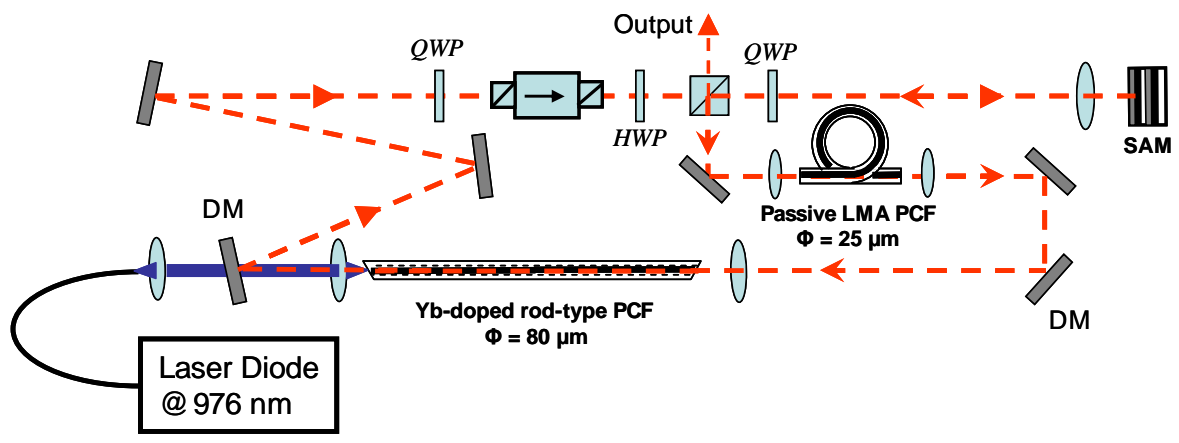

Fig. 1: Experimental setup of the chirped-pulse fiber oscillator

\section{Experimental Results}

The laser produces a stable self-starting pulse train at a repetition rate of $15.5 \mathrm{MHz}$. Preliminary results show that $13 \mathrm{~W}$ of average power, corresponding to $830 \mathrm{~nJ}$, could be achieved. The laser delivers highly-chirped picosecond pulses with about $10 \mathrm{~nm}$ spectral widths. The typical laser outputs obtained for an average power of $13 \mathrm{~W}$ are shown in Fig. 2. The spectrum is centered around $1028 \mathrm{~nm}$ wavelength with a spectral width (FWHM) of $9.3 \mathrm{~nm}$. The optical spectrum presents a typical steep edged shape with a parabolic top. The autocorrelation trace measurement shows that the pulse duration is $27.6 \mathrm{ps}$ assuming a sech ${ }^{2}$ pulse shape [Fig. 2(b)]. The output pulses are extra-cavity dechirped to 550 fs duration using transmission gratings [Fig. 3]. This duration is 1.7 times higher than the transform-limited duration calculated from the spectrum (327 fs). The corresponding peak power is higher than 1.2 MW. The radiofrequency measurements reveal a good amplitude stability of the output pulse train. The pulse to pulse energy fluctuations are estimated to be less that $0.04 \%$. We note that the current laser performances are only limited by the pump power.
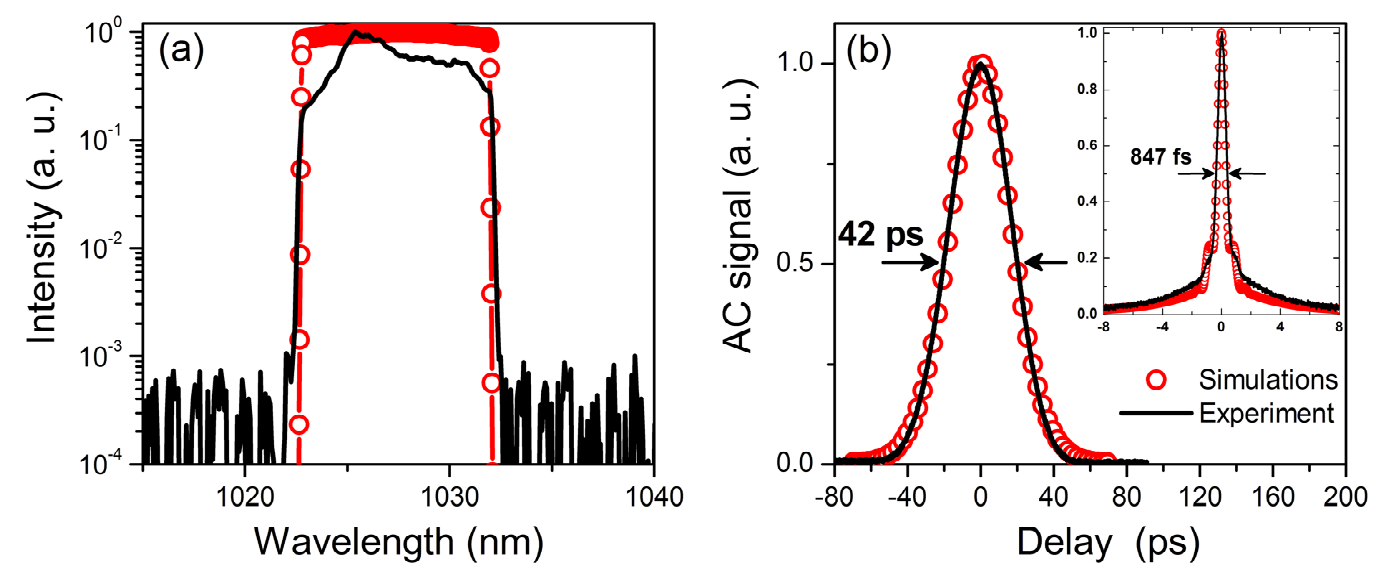

Fig. 2: Typical output spectrum and (b) output pulse autocorrelation. Inset, dechirped pulse autocorrelation

The output pulse parameters are less sensitive to the output coupling ratio compared to the laser configuration based on resonant SESAMs [10]. This suggests that pulse shaping is dominated by the saturable absorber nonlinearity with negligible contribution from nonlinear polarization evolution. To check this hypothesis and study the laser potentialities for energy scaling, numerical simulations were performed considering the laser set-up shown on Fig. 1. Pulse propagation along the gain fiber is described by the extended nonlinear Schrödinger equation which includes the effects of dispersion, Kerr nonlinearity and saturated gain with a finite bandwidth of $45 \mathrm{~nm}$. Absorption of the SAM is described by the rate equation model with a relaxation time of $500 \mathrm{fs}$. The results obtained for accurate laser parameters are depicted on Fig. 2. Numerical results are in good agreement with experiments and reveal that pulse shaping is dominated by the amplitude modulation provided by the SAM in combination with gain filtering, see Fig. 3. The pulse lengthening happening along the passive fiber is partially compensated by the spectral narrowing 
happening along the gain fiber. This behavior is governed by the high net cavity dispersion. Indeed, insertion of a long passive fiber enables temporal pulse broadening just behind the gain fiber. This contributes to increase the amplitude modulation induced by gain filtering which acts on highly-chirped pulses. The complete pulse selfconsistency is insured by the contribution of the SAM. We note that the monotonic evolution along the passive fiber indicates that the cumulated frequency-chirp is mainly linear. The negligible SPM endured along the gain fiber allows maintaining the linear chirp which is partially removed by the SAM action. The temporal evolution along the cavity resembles that of the self-similar laser [3]. The parabolic-top output spectrum is an additional signature of self-similar pulse evolution (Fig. 2). Moreover, numerical simulations predict that stable pulse solutions do exist for more than $10 \mu \mathrm{J}$ intra-cavity pulse energy, revealing a great potential for energy scaling.

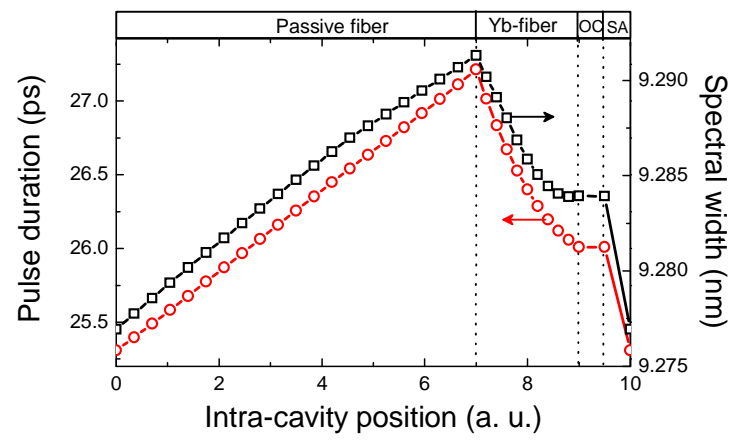

Fig. 3 : Pulse evolution inside the cavity in the spectral and temporal domains.

\section{Conclusion}

In conclusion, we have demonstrated the generation of highly-stretched 27 ps pulses with up to $830 \mathrm{~nJ}$ energy from a highly normal dispersion laser featuring large core microstructured fibres. The output pulses are externally dechirped to 550 fs duration with 1.2 MW peak power. Numerical simulations reveal that pulse shaping is dominated by the gain filtering action in combination with the amplitude modulation in the SAM. More details on the energy scaling potential of this source will be discussed in this communication.

\section{Acknowledgement}

This work is supported by the Inter Carnot-Fraunhofer program under project APUS and the French Agency for Research under project OFFEMET. We acknowledge support from the Conseil Régional de Haute Normandie.

\section{References}

[1] C. Baer, C. Kränkel, C. Saraceno, O. Heckl, M. Golling, R. Peters, K. Petermann, T. Südmeyer, G. Huber and U. Keller, "Femtosecond thindisk laser with $141 \mathrm{~W}$ of average power," Opt. Lett 35, 2302-2304 (2010).

[2] K. Tamura, E. P. Ippen, H. A. Haus, and L. E. Nelson, "77-fs pulse generation from a stretched-pulse mode-locked all-fiber ring laser," Opt. Lett. 18, 1080-1082 (1993).

[3] F. Ö. Ilday, J. R. Buckley, W. G. Clark, and F. W. Wise, "Self-Similar Evolution of Parabolic Pulses in a Laser," Phys. Rev. Lett. 92, 213902 (2004).

[4] L. M. Zhao, D. Y. Tang, and J. Wu, "Gain-guided soliton in a positive group-dispersion fiber laser," Opt. Lett. 31, 1788-1790 (2006).

[5] A. Chong, J. Buckley, W. Renninger, and F. Wise, "All-normal dispersion femtosecond fiber laser," Opt. Express 14, 10095 (2006).

[6] C. Lecaplain, B. Ortaç, and A. Hideur, "High-energy femtosecond pulses from a dissipative soliton fiber laser," Opt. Lett. 34, 3731-3733 (2009).

[7] S. Lefrançois, K. Kieu, J. Deng, J. D. Kafka and F. W. Wise "Scaling of dissipative soliton fiber lasers to megawatt peak powers by use of large-area photonic crystal fiber", Opt. Lett. 35, 1569-1571 (2010).

[8] M. Baumgartl, B. Ortaç, C. Lecaplain, A. Hideur, J. Limpert and A. Tünnermann, "Sub-80 fs dissipative soliton large mode area fiber laser", Opt. Lett. 35, 2311-2313 (2010).

[9] B. Ortaç, M. Baumgartl, J. Limpert, and A. Tünnermann, "Approaching microjoule level pulse energy with mode-locked femtosecond fiber lasers," Opt. Lett. 34, 1585-1587 (2009).

[10] C. Lecaplain, B. Ortaç, G. Machinet, J. Boullet, M. Baumgartl, T. Schreiber, E. Cormier, and A. Hideur, "High-energy femtosecond photonic crystal fiber laser," Opt. Lett. 35, 3156-3158 (2010). 